\title{
Improvement of molecular-genetic diagnostics of the most common skeletal dysplasias
}

\author{
Kotysova $\mathrm{L}^{1,2}$, Mattosova $\mathrm{S}^{1,2}$, Chandoga $\mathrm{J}^{1}$ \\ Institute of Medical Biology, Genetics and Clinical Genetics, Comenius University Faculty of Medicine \\ and University Hospital Bratislava, Bratislava, Slovakia. livia.kotysova@gmail.com
}

\begin{abstract}
OBJECTIVES: The aim of this study was to take the molecular-genetic methods for detection of the most frequent mutations in patients suspected for achondroplasia $(\mathrm{ACH})$ and hypochondroplasia $(\mathrm{HCH})$ into the routine practice. BACKGROUND: Both disorders are usually caused by de novo gain-of-function type mutations in FGFR3 gene encoding the fibroblast growth factor receptor 3 , which plays an important role in the metabolism of connective tissues. More than $99 \%$ of $\mathrm{ACH}$ cases are caused by the glycine-to-arginine substitution at codon 380 and about $70 \%$ of $\mathrm{HCH}$ cases result from the asparagine-to-lysine/-serine/-threonine substitutions at codon 540 in the consequence of the four different possible nucleotide changes occurred at the same codon.

METHODS: Exons 10 and 13 of theFGFR3 gene were analysed by PCR-RFLP and sequencing analysis. The exon 13 sequencing was necessary for mutation type specification.

RESULTS: We confirmed the diagnosis of $\mathrm{ACH}$ due to $1138 \mathrm{G} \rightarrow \mathrm{A}$ transition in 7 patients and we identified $1620 \mathrm{C} \rightarrow \mathrm{A}$ transversion responsible for $\mathrm{HCH}$ in 2 patients.

CONCLUSION: Due to serious limitations in recently used methods, we had to modify the molecular-genetic di-agnostics approach. We developed the reliable diagnostics and made it available for achondroplasia and hypochondroplasia suspected patients (Tab. 1, Ref. 5, Ref. 17). Text in PDF www.elis.sk.

KEY WORDS: achondroplasia, hypochondroplasia, FGFR3 gene, 1138 G-to-A transition, 1620 C-to-A tranversion.
\end{abstract}

\section{Introduction}

Achondroplasia (ACH, MIM 100800) and Hypochondroplasia (HCH, MIM 146000) are the common types of skeletal dysplasias with the prevalence between $1: 30000$ and $1: 10000$ predominantly characterised by short stature and dwarfism. They are inherited in an autosomal-dominant manner. Clinical features of achondroplasia are associated with enlarged head, depressed nasal bridge, midfacial hypoplasia, proximal shortening of the extremities, short stubby trident hands, genu varum, limitation of elbow extension, megalencephaly, lordotic lumbar spine, prominent buttocks, protuberant abdomen and final adult height of approximately $125 \mathrm{~cm}$ for males and $120 \mathrm{~cm}$ for females $(1,2)$. Hypochondroplasia is characterised by clinical manifestations milder than in $\mathrm{ACH}$ with the average height of adults $-146 \mathrm{~cm}$ in males and $138 \mathrm{~cm}$ in females, macrocephaly, limitation of elbow extension, varus deformity of the lower extremities, lumbar lordosis, narrowing of the spinal canal, seldom spinal stenosis and mild to moderate

${ }^{1}$ Institute of Medical Biology, Genetics and Clinical Genetics, Comenius University Faculty of Medicine and University Hospital Bratislava, Bratislava, Slovakia, and ${ }^{2}$ Department of Genetics, Faculty of Natural Sciences of Comenius University Bratislava, Bratislava, Slovakia

Address for correspondence: L. Kotysova, MSc, Institute of Medical Biology, Genetics and Clinical Genetics, Comenius University Faculty of Medicine and University Hospital Bratislava, Mickiewiczova 13, SK-813 69 Bratislava, Slovakia.

Phone: +421.911338618 mental retardation (3). Both disorders results from recurrent missense mutations in FGFR3 gene, which spans over 18 exons and lies within the $4 p 16.3$ region (4). Gene encodes the member of the family of tyrosine kinase (TK) transmembrane receptors (FGFR1, FGFR2, FGFR3 and FGFR4). They bind fibroblast growth factors with low capacity and high affinity. Mutations cause receptor activation and inhibition of chondrocyte proliferation, what leads to abnormalities of epiphyseal plate function and bone tissues growth.

FGFR3 receptor is highly homologous to other members of TK family and consists of three extracellular immunoglobulin-like FGF-binding domains (Ig I-III), one hydrophobic transmembrane (TM) domain and two cytoplasmic tyrosine kinase sub-domains TK1 and TK2 responsible for the catalytic activity. Typical features for FGFRs are the unique patterns of expression during embryogenesis, suggesting their different functions of FGFs mediating (5).

More than $99 \%$ of achondroplasia is caused by the heterozygous glycine-to-arginine substitution at codon 380 in the transmembrane domain. About $98 \%$ of this substitution cases are based on the Gto-A transition and about $1 \%$ of cases on the G-to-C transversion at nucleotide 1138 (6). All new mutations occur on the paternal allele, suggesting an increased mutability of FGFR3 during spermatogenesis and are associated with an increased paternal age (7). Homozygosity or compound heterozygosity states are often manifest with a more severe phenotype and are lethal in the perinatal period (8).

The most common mutation detected in about $65 \%$ cases of hypochondroplasia is the $\mathrm{N} 540 \mathrm{~K}$ amino-acid substitution in the TK1 domain that can lead to the disruption of the $\alpha$-helical structure 


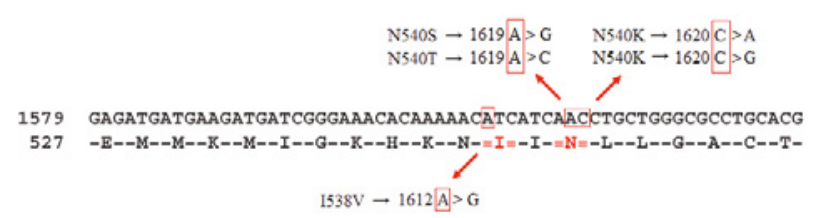

Fig. 1. The position of four possible substitutions occurred in codon 540 and of I538V amino-acid change at exon 13 that lead to HCH.

of this hydrophobic region. The first discovered mutation responsible for this quite frequent amino-acid substitution was $1620 \mathrm{C} \rightarrow \mathrm{A}$ (8) followed by finding a family-type $1620 \mathrm{C} \rightarrow \mathrm{G}$ mutation leading to the same amino-acid substitution (asparagine-to-lysine) (9). In 1998, a 35-year-old man was found with the substitution of one nucleotide before nucleotide 1620 , the $1619 \mathrm{~A} \rightarrow \mathrm{C}$ transversion, leads to the arginine-to-threonine amino-acid substitution (N540T) (10). Two years later, another predictable mutation located at the same region was found. It was the $1619 \mathrm{~A} \rightarrow \mathrm{G}$ transition leading to the arginine-to-serine substitution (N540S) (11). Finally, four possible nucleotides changes were discovered, which can lead to three different amino-acids substitutions that arise at the same codon (Fig. 1). At present, it is evident that $\mathrm{HCH}$ can be caused also by other substitutions like N328I, I538V, K650N/Q, but many of them are private mutations only with single family recurrence $(12,13,14)$.

Though molecular-genetic and biochemical base of $\mathrm{ACH}$ and $\mathrm{HCH}$ is known for more than 20 years, no effective therapy for bone growth correcting can be offered to patients. Nowadays, some new strategies for therapy are in development. Both disorders result from an increased signal transduction from the mutant receptor. Therefore, therapeutic strategies are focused on reducing these signals. The first tested therapeutics, like kinase inhibitors, directly targeting FGFR3 are in the preclinical phase of testing. For example binding of C-type natriuretic peptide to its receptor, natriuretic-peptide receptor B (NPR-B), inhibits signal transduction pathways downstream of FGFR3 $(15,16)$.

\section{Material and methods}

\section{Patients}

Our study involved patients from all regions of Slovakia. Blood samples of patients with suspected diagnoses of ACH and $\mathrm{HCH}$ based on clinical and radiological examinations were sent to our Institute in period of last three years. Before the year 2011, molecular-genetic diagnostics was not available in Slovakia. All subjects signed an informed written consent. DNA was extracted from peripheral blood cells or from amniotic fluid cells using MN
NucleoSpin Blood- Mini according to the manufacturer's protocol (Macherey-Nagel).

\section{Mutation analyses}

Genomic DNA was amplified using primer sets common for both methods, for PCR-RFLP and sequencing analyses. Primers flanking the intron-exon boundaries were designed using Primer 3 software v. 0.4.0 and checked by SNPCheck v3 and NCBI/Blast softwares. PCR amplification of FGFR3 exons 10 and 13 was performed under the following conditions. Exon 10 was amplified using 5x Phusion ${ }^{\circledR}$ GC buffer (Thermo Fisher Scientific), 0.2 $\mathrm{mM}$ dNTPs, $3 \%$ DMSO, $0.75 \mathrm{M}$ Betaine, $0.5 \mu \mathrm{M}$ of each primer and $0.02 \mathrm{U} / \mu 1$ of Phusion ${ }^{\circledR}$ High-Fidelity DNA polymerase in a reaction volume of $20 \mu$ l. Exon 13 was amplified using $2 x$ PCR Master Mix (Thermo Fisher Scientific), 3\% DMSO and $0.3 \mu \mathrm{M}$ of each primer in a reaction volume of $25 \mu$ l. Primer sequences, amplification conditions for Mastercycler EPGradient (Eppendorf ${ }^{\circledR}$ ) and product lengths were summarised in Table 1.

In accordance with the known facts that mutations create novel restriction sites, screening for G380R mutations in exon 10 was performed by digestion with $3 \mathrm{U}$ of restriction enzymes $-S f C I$ and MspI (1). $1138 \mathrm{G}>\mathrm{A}$ transition creates the novel restriction site for $S f C I$ (recognition sequence 5'-C ${ }^{\mathbf{v}}$ TRYAG-3') and $1138 \mathrm{G}>\mathrm{C}$ transversion for MspI (recognition sequence 5'- ' $\mathrm{CGG}$-3'). The digested products were separated on the Spreadex ${ }^{\circledR}$ EL 800 Mini gel (Elchrom Scientific) with working separation range of 60-800bp and optimal range of 200-500bp under conditions: $110 \mathrm{~V}$ on $20^{\circ} \mathrm{C}$ for 2 hours and 45 minutes. Visualisation was made by solution of GelRed ${ }^{\mathrm{TM}}$ Nucleic Acid Gel Stain and $\mathrm{NaCl}$.

We found that all mutations in codon 540 abolish a restriction site for enzyme, which recognizes non-mutated standard sequence. PCR product of exon 13 was at first digested with $4 \mathrm{U}$ of $B f u A I$ (recognition sequence $5^{\prime}$-ACCTGC(N) $4^{\mathbf{\nabla}}-3^{\prime}$ ') to distinguish between pathological and normal allele. The digested products were separated on a $2 \%$ agarose gel. BfuAI is an isoschizomer of BspMI and cleaves target sequences more efficiently. It belongs to a group of enzymes, which requires two copies of its recognition sequence for effective cleavage and furthermore needs to interact with the DNA $>6$ bp upstream and $>10$ bp downstream of the recognition site. Therefore, after the first mutation screening by PCR-RFLP, it was necessary to made a sequencing analysis.

For verification of detected G380R mutations and for identification of 540 codon mutations, a sequencing analysis has to be performed. PCR products were enzymaticaly purified using thermosensitive alkaline phosphatase FastAP ${ }^{\mathrm{TM}}$ and Exonuclease I (Thermo Fisher Scientific) and directly sequenced. Sequencing analysis was

Tab. 1. Primers for amplification and sequencing of genomic DNA and conditions for PCR amplification.

\begin{tabular}{|c|c|c|c|}
\hline Primer location & Primer sequence $5^{\prime} \longrightarrow 3^{\prime}$ & Amplification conditions & Product size $(\mathrm{bp})$ \\
\hline FGFR3ex10F & CAGGCCAGGCCTCAACGCCC & \multirow{2}{*}{$\begin{array}{l}98^{\circ} \mathrm{C} \times 1 \mathrm{~min} \\
{\left[98^{\circ} \mathrm{C} \times 10 \mathrm{~s} ; 72^{\circ} \mathrm{C} \times 30 \mathrm{~s}\right] \times 27 \text { cycles }} \\
72^{\circ} \mathrm{C} \times 7 \mathrm{~min}\end{array}$} & \multirow[t]{2}{*}{$908 \mathrm{bp}$} \\
\hline$\overline{\text { FGFR3ex10R }}$ & GACGAAGAGTGTCACCCACA & & \\
\hline FGFR3ex13F & CCGTGAAGATGCTGAAAGGT & \multirow{2}{*}{$\begin{array}{l}95 \pm{ }^{\circ} \mathrm{C} \times 4 \mathrm{~min} \\
{\left[95^{\circ} \mathrm{C} \times 30 \mathrm{~s} ; 59{ }^{\circ} \mathrm{C} \times 30 \mathrm{~s} ; 72{ }^{\circ} \mathrm{C} \times 30 \mathrm{~s}\right] \times 30 \text { cycles }} \\
72{ }^{\circ} \mathrm{C} \times 7 \mathrm{~min}\end{array}$} & \multirow[t]{2}{*}{$395 b p$} \\
\hline FGFR3ex13R & CTTGCAGGTGTCGAAGGAGT & & \\
\hline
\end{tabular}




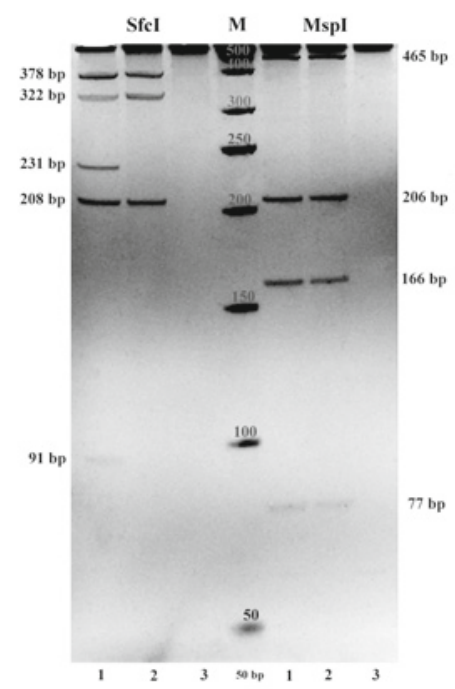

Fig. 2. PCR-RFLP analyses; the results of $S f c I$ and $M s p I$ digestions. In SfCI digestion: lane 1, sample with 1138G $>$ A transition in heterozygous form; lane 2, patient without mutation (wild-type sample); lane 3, control sample without DNA. In MspI digestion: same patients as in SfcI digestion were analysed, both without detected G-to-C transversion.

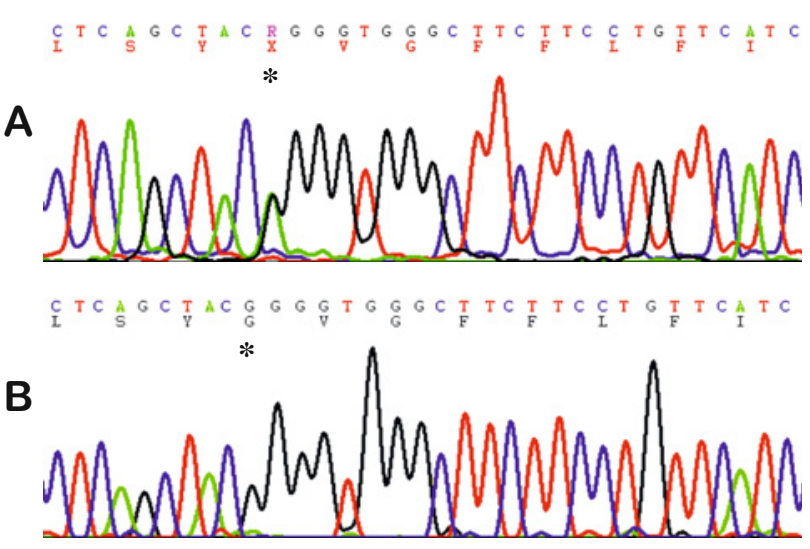

Fig. 3. DNA sequence analysis of exon 10 of the FGFR3 gene. A: The Gto-A transition in heterozygous state resulting in the glycine-to-arginine exchange on one allele and is present in 7 of our patients. B: Mutation absence in unaffected individuals. Sign (*) indicates mutation position.

performed with ABI 3100 Genetic Analyser using BigDye ${ }^{\mathrm{R}}$ Terminator v3.1 Cycle Sequencing kit, following the manufacturer's instructions. Data were analysed by Chromas 2.2 (Technelysium Pty Ltd., Australia) and Vector NTI 11.5 (Informax) and compared to reference sequence from GenBank (NM_000142.4).

\section{Results}

In the period of three years, we confirmed the diagnosis of $\mathrm{ACH}$ and $\mathrm{HCH}$ in 9 patients from 25 requested probands. 7 patients carried the G380R mutation in the position $1138 \mathrm{G}>\mathrm{A}$ transition leading to $\mathrm{ACH}$ phenotype and 2 carried the $\mathrm{N} 540 \mathrm{~K}$ mutation in the position $1620 \mathrm{C}>\mathrm{A}$ transversion - leading to $\mathrm{HCH}$ phenotype.

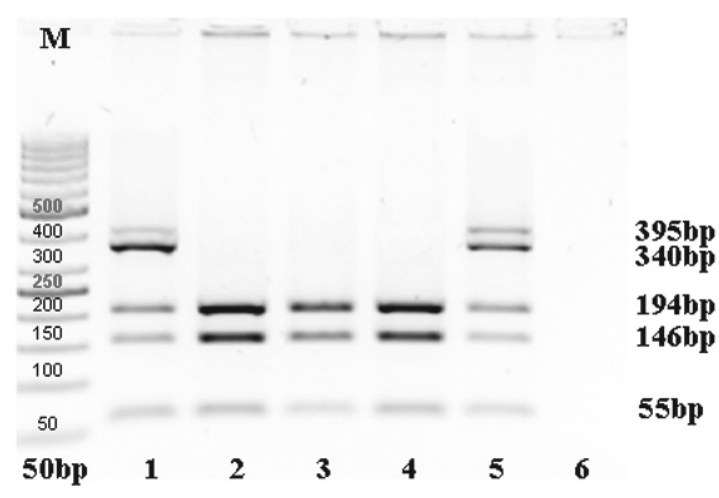

Fig. 4. PCR-RFLP analyses; the result of $B f u A I$ digestion. Lanes 1 and 5, samples with 540 codon's mutation in the heterozygous form; lanes 2 to 4, patients without 540 codon's mutation (wild-type samples); lane 6, control sample without DNA. Restriction fragment with the length of 395bp, in the patients' samples with heterozygous form of mutation, was result of an incomplete non-effective digestion due to presence of only one restriction site.

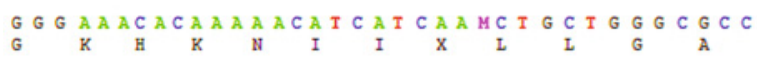

A


B

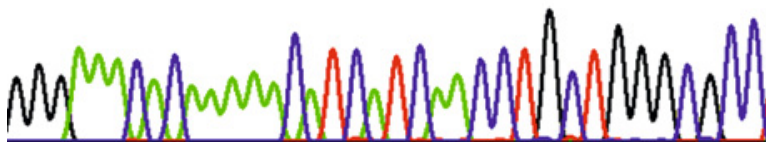

Fig. 5. DNA sequence analysis of exon 13 of the FGFR3 gene and mutation specification. A: The C-to-A transversion in heterozygous state resulting in the asparagine-to-lysine exchange, which is present in our 2 patients. B: Mutation absence in unaffected individuals. Sequences shown are the proportion of exon 13's chromatogram with corresponding amino acid single-letter codes. Sign (*) indicates mutation position.

\section{Mutation analyses for ACH}

Previous published methods for molecular-genetic diagnostics (17) was based on amplification of very short fragment (164bp) containing only one restriction site located in hot spot mutation codon. For the judgement of a digestion effectivity it is recommended to set other digestion control sites except the site of interest. Therefore, we set up amplification of larger product to avoid the false-negative results. The $908 \mathrm{bp}$ PCR product is subsequently digested by two restriction enzymes. After $S f c I$ digestion resulted in fragments of 322, 378, 208bp in wild-types and in fragments of $\mathbf{9 1}$, $231,378,208$ bp if the mutation $1138 \mathrm{G}>\mathrm{A}$ is present. If the novel restriction site is create by the $\mathrm{G}$-to- $\mathrm{C}$ transversion, the fragment sizes after MspI digestion will be 465, 206, 160, 77bp in wild type and 95, 370, 206, 160, 77bp in case of mutation presence (Fig. 2).

Sequencing of FGFR3 exon 10 was applied in aim to verificate of RFLP analysis findings (Fig. 3). 


\section{Mutation analyses for $\mathrm{HCH}$}

In two patients, the restriction analysis of the $395 \mathrm{bp}$ amplified PCR product resulted in abolition of the digestion site for $B f u A I$, demonstrating the presence of a closely unspecified mutation in codon 540 in heterozygous state. The fragment sizes after the digestion were 194, 146, 55bp in wild-type and 340,55bp in case of mutation presence, due to the restriction site abolishment (Fig. 4).

Sequencing analysis had to be done in all cases, in which the 540 codon closely unspecified mutation was identified by PCRRFLP to determine the mutation type (Fig. 5).

\section{Discussion}

Recently, it was supposed that there are only few causal mutations involved in phenotypes of achondroplasia and hypochondroplasia. According to present data, the $\mathrm{N} 540 \mathrm{~K}$ substitution should be a cause of $\mathrm{HCH}$ in about $65 \%$ of patients (8). For ACH, the assumption was much more rigid, because it was known that as much as $98 \%$ of positive cases carried the heterozygous amino-acid substitution of G380R. It indicates that this sequence region is the most sensitive area for germline mutation in the entire FGFR3 gene (6). To date, more new causal mutations were identified. Only two new mutations have been found to be responsible for ACH and both of them lied within the transmembrane domain, as so as G380R. Seventeen new mutations have been found to lead to $\mathrm{HCH}(2)$. However, according to present knowledge codons 380 and 540 are the most important mutation hot-spots.

We combined two methods PCR-RFLPs and sequencing analysis for diagnostics purposes.

We consider the sequencing analysis as the only one possible and reliable way to distinguish between mutations types that can occurred within the 540 codon in exon 13 of the FGFR3 gene. Despite the restriction enzyme advantage, PCR-RFLP method is in that case highly uninformative. Our findings obtained using these techniques are in agreement with previous reports. We identified $1620 \mathrm{C}>$ A transversion in two patients with typical hypochondroplasia phenotype.

Every our patient with a confirmed diagnosis of achondroplasia had the same mutation type $-1138 \mathrm{G}>\mathrm{A}$ transition, which is the single most mutable nucleotide in the human genome. This most frequent FGFR3 substitution (G380R) induces constitutive activation of the receptor producing by ligand binding. In consequence to this molecular phenomenon, a prolonged signalling at the cell surface is observed (1). All ACH patients had typical clinical findings with predomination of rhizomelic limbs shortening, macrocephaly and facial dysmorphy. We confirmed the supposed clinical diagnosis at early childhood period. Four of our patients were actually newborns, 2 were nursling and the oldest one was at the age of 4 and this pointed to the importance of the prompt and early determination of diagnosis.

Unlikely, clinical diagnosis is often not made during infancy and many affected children are present to specialists for failure to grow more later in childhood. Prompt and definitive diagnosis of both disorders could be made also prenatal and very early postnatal and will have important implications for prognosis, clinical management and genetic counselling of other family members. The combination of two molecular genetic methods allows a reliable diagnosis and becomes available for patients from Slovakia.

\section{References}

1. Bellus GA, Hefferon TW, Ortiz de Luna RI, Hecht JT, Horton WA, Machado M, Kaitila I, McIntosh I, Francomano CA. Achondroplasia is defined by recurrent G380R mutations of FGFR3. Am J Hum Genet 1995; 56 (2): 368-373.

2. Baujat G, Legeai-Mallet L, Finidori G, Cormier-Daire V, Le Merrer M. Achondroplasia. Best Pract Res Clin Rheumatol 2008; 22 (1): 3-18.

3. Bellus GA, McIntosh I, Szabo J, Aylsworth A, Kaitila I, Francomano CA. Hypochondroplasia: molecular analysis of the fibroblast growth factor receptor 3 gene. Ann N Y Acad Sci 1996; 785: 182-187.

4. Le Merrer M, Rousseau F, Legeai-Mallet L, Landais JCh, Pelet A, Bonaventure J, Sanak M, Weissenbach J, Stoll C, Munnich A, Maroteaux P. A gene for achondroplasia - hypochondroplasia maps to chromosome 4p. Nat Genet 1994; 6: 318-321.

5. Johnson DE, Williams LT. Structural and functional diversity in the FGF receptor multigene family. Adv Cancer Res 1993; 60: 1-41.

6. Rousseau F, Bonaventure J, Legeai-Mallet L, Pelet A, Rozet JM, Maroteaux P, Le Merrer M, Munnich A. Mutations in the gene encoding fibroblast growth factor receptor-3 in achondroplasia. Nature 1994; 371 (6494): 252-254.

7. Wilkin DJ, Szabo JK, Cameron R, Henderson S, Bellus GA, Mack ML, Kaitila I, Loughlin J, Munnich A, Sykes B, Bonaventure J, Francomano CA. Mutations in fibroblast growth-factor receptor 3 in sporadic cases of achondroplasia occur exclusively on the paternally derived chromosome. Am J Hum Genet 1998; 63 (3): 711-716.

8. Bellus GA, McIntosh I, Smith EA, Aylsworth AS, Kaitila I, Horton WA, Greenhaw GA, Hecht JT, Francomano CA. A recurrent mutation in the tyrosine kinase domain of fibroblast growth factor receptor 3 causes hypochondroplasia. Nat Genet 1995; 10 (3): 357-359.

9. PrinosP,CostaT,SommerA,KilpatrickMW,TsipourasP.AcommonFGFR3 gene mutation in hypochondroplasia. Hum Mol Genet 1995; 4 (11): 2097-2101.

10. Deutz-Terlouw PP, Losekoot M, Aalfs CM, Hennekam RC, Bakker E. Asn540Thr substitution in the fibroblast growth factor receptor 3 tyrosine kinase domain causing hypochondroplasia. Hum Mutat 1998; Suppl 1: S62-65.

11. Mortier G, Nuytinck L, Craen M, Renard JP, Leroy JG, de Paepe A. Clinical and radiographic features of a family with hypochondroplasia owing to a novel Asn540Ser mutation in the fibroblast growth factor receptor 3 gene. J Med Genet 2000; 37 (3): 220-224.

12. WinterpachtA, Hilbert K, Stelzer C, Schweikardt T, Decker H, SegererH, Spranger J, Zabel B. A novel mutation in FGFR-3 disrupts a putative N-glycosylation site and results in hypochondroplasia. Physiol Genomics 2000; 2 (1): 9-12.

13. Grigelioniené G, Hagenäs L, Eklöf O, Neumeyer L, Haereid PE, Anvret M. A novel missense mutation Ile538Val in the fibroblast growth factor receptor 3 in hypochondroplasia. Hum Mutat 1998; 11 (4): 333-340.

14. Bellus GA, Spector EB, Speiser PW, Weaver CA, Garber AT, Bryke CR, Israel J, Rosengren SS, Webster MK, Donoghue DJ, Francomano CA. Distinct missense mutations of the FGFR3 lys650 codon modulate receptor kinase activation and the severity of the skeletal dysplasia phenotype. Am J Hum Genet 2000; 67 (6): 1411-1421.

15. Laederich MB, Horton WA. Achondroplasia: pathogenesis and implications for future treatment. Curr Opin Pediatr 2010; 22 (4): 516-523.

16. Lorget F, Kaci N, Peng J, Benoist-Lasselin C, Mugniery E, Oppeneer T, Wendt DJ, Bell SM, Bullens S, Bunting S, Tsuruda LS, O’Neill CA, Di Rocco F, Munnich A, Legeai-Mallet L. Evaluation of the therapeutic potential of a CNP analog in a Fgfr3 mouse model recapitulating achondroplasia. Am J Hum Genet 2012; 91 (6): 1108-1114.

17. Shiang R, Thompson LM, Zhu YZ, Church DM, Fielder TJ, Bocian M, Winokur ST, Wasmuth JJ. Mutations in the transmembrane domain of FGFR3 cause the most common genetic form of dwarfism, achondroplasia. Cell 1994; 78 (2): 335-342. Accepted March 8, 2015. 